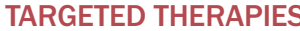

\section{One step closer to drugging p53}

A new phase I trial has shown that a small, 28-amino-acid peptide called p28, which stabilizes p53 (encoded by TP53) to protect it from degradation, is welltolerated in patients with advanced solid tumours. The work-a first-in-class, firstin-human trial-also demonstrated that some tumour control could be achieved.

Approximately $50 \%$ of all tumours harbour mutations in the tumour suppressor gene TP53. These tumours can also lose feedback control of levels of the p53 protein, which is normally mediated through the endogenous regulator MDM2 in a negative-feedback loop. The peptide p28 acts by binding to p53 at its DNAbinding domain, which stabilizes the protein and prevents its degradation. This stabilization leads to an increase in p53, which leads to an increase of its downstream targets (such as p21 and p27) and inhibition of the cell cycle at G2/M.

The phase I study was initiated after favourable preclinical study data, and made use of the accelerated titration design, which meant that patients who did not experience a dose-limiting toxicity could be kept on study to receive the next dose (intrapatient escalation). Included in the study were 15 patients who had a range of advanced cancers, including melanoma, colon cancer and pancreatic cancer. Doses of 0.83-4.16 mg per kg body weight were delivered intravenously three times per week for 4 weeks, which was followed by a 2 -week rest before dose escalation.

No patient experienced a doselimiting toxic event, and the maximum tolerated dose could not be determined. Importantly, seven patients demonstrated extended periods (7-61 weeks) of stable disease and three patients had a partial response that lasted 44-125 weeks. One patient had a complete response that lasted $>2$ years. These promising results are due to be followed up with an efficacy trial.

\section{Mina Razzak}

Original article Warso, M. A. et al. A first-in-class, first-inhuman, phase I trial of p28, a non-HDM2-mediated peptide inhibitor of $p 53$ ubiquitination in patients with advanced solid tumours. Br. J. Cancer doi:10.1038/bjc.2013.74 